\title{
METABOLIC DISTURBANCES IN THE PATIENTS WITH ACUTE ISCHEMIC STROKE
}

\author{
Daniela Y. Arabadzhieva, \\ Ara G. Kaprelyan, \\ Zhaneta T. Georgieva', \\ Zdravko D. Slavov', \\ Aleksandra Zh. Tsukeva
}

First Clinic of Neurology, ${ }^{1}$ Department of Propedeutics of Internal Medicine, St. Marina University Hospital of Varna

${ }^{2}$ Chernorizets Hrabar Varna Free University,

Varna

\author{
Corresponding Author: \\ Daniela Y. Arabadzhieva \\ First Clinic of Neurology \\ St. Marina University Hospital of Varna \\ 1, Hristo Smirnenski blvd \\ Varna, 9010 \\ Bulgaria \\ e-mail:d.arabadzhieva@abv.bg
}

Received: October 30, 2014

Revision received: December 14, 2014 Accepted: December 19, 2014

\section{Summary}

The purpose of this study was to analyze the disturbances of glucose, lipid and protein metabolism in acute ischemic stroke patients. A total of 258 patients (mean age $70.9 \pm 7.22$ years, range $49-92$ years) were studied. The following parameters were examined: blood glucose, triglycerides, total cholesterol, HDL-cholesterol, LDLcholesterol, creatinine, and urea. Data were statistically processed by variation and correlation analysis. Our results demonstrated abnormal values of these laboratory parameters in most patients. The mean concentrations of triglycerides, total cholesterol and LDL-cholesterol were higher in females, while these of blood glucose were higher in males, especially in the age group between 76 and 80 years. Pearson's correlation coefficient was highest between the variables 'total cholesterol' and 'LDLcholesterol' ( $\mathrm{r}=0.797)$ but moderate - between some of the rest variables such as 'creatinine' and 'urea' $(\mathrm{r}=0.575)$; 'total cholesterol' and 'triglycerides' $(\mathrm{r}=0.565)$; 'total cholesterol' and 'urea' (r=0.428); 'triglycerides' and 'urea' $(\mathrm{r}=0.370)$ and 'LDL-cholesterol' and 'urea' $(\mathrm{r}=0.301)$. In conclusion, the metabolic disorders are relatively common among acute ischemic stroke patients and play a specific role as risk factors for this disease. These parameters should regularly be controlled within the outpatient practice in order to warrant an effective prevention of acute ischemic stroke.

Key words: acute ischemic stroke, blood glucose, lipids, creatinine, urea

\section{Introduction}

Metabolic disorders within the metabolic syndrome or separately as specific abnormalities of glucose, lipid and protein metabolism represent an important precondition for the development of cardiovascular and cerebrovascular diseases worldwide. Impaired glucose tolerance is often present in patients with ischemic stroke (IS) and doubles the risk of this disease. Dyslipidemia, elevated low-density lipoprotein cholesterol (LDL-C) and arterial hypertension are important but modifiable risk factors for adult coronary artery disease (CAD) and IS as well. Elevated total cholesterol (TC), high 
LDL-C and low high-density lipoprotein cholesterol (HDL-C) are modifiable risk factors for IS. There is substantial evidence that hypertriglyceridemia might be an independent risk factor for IS [1].

The purpose of this study was to analyze the disturbances of glucose, lipid and protein metabolism in adult acute IS (AIS) patients.

\section{Materials and Methods}

A total of 258 AIS patients (mean age $70.59 \pm 7.22$ years, range 49-92 years) were studied. There were 129 males aged $69.60 \pm 7.80$ years (range 49 87 years) and 129 females aged $71.59 \pm 6.65$ years (range 58-92 years). The diagnosis of AIS was confirmed by Doppler sonography and computed tomography of the cerebral circulation. The following parameters were examined: blood glucose, triglycerides, TC, LDL-C, HDL-C, creatinine, and urea. Data were statistically processed by variation (ANOVA as $t$-criterion was considered significant if $\mathrm{p}<0.05$ ) analysis and correlation (Pearson's coefficient) analysis as well. SPSS, version 13.0 software was applied.

\section{Results}

Distribution of patients by age is indicated on Table 1.

Age-related dependences concerning the parameters of glucose and lipid metabolism in male AIS patients are presented on Table 2, and those in female ones - on Table 3.

Our results demonstrated abnormal values of these laboratory parameters in most of the patients. The mean blood glucose value in males aged $\leqslant 65$ years was statistically significantly higher than that in females $(t=2.44 ; \mathrm{p}<0.01)$. The difference between the number of the male patients with pathologically higher blood glucose concentrations and that of the patients with normal ones was statistically significant $(t=3.33$; $\mathrm{p}<0.01)$.

The mean concentrations of triglycerides, TC and LDL-C were higher in the females, while these of blood glucose were higher in the males, especially in the 76-80 age group.

Table 1. Age distribution of AIS patients

\begin{tabular}{lllllll}
\hline \multirow{2}{*}{ Age (years) } & males & \multicolumn{3}{c}{ females } & \multicolumn{2}{c}{ total } \\
\cline { 2 - 7 } & $\mathrm{n}$ & $\%$ & $\mathrm{n}$ & $\%$ & $\mathrm{n}$ & $\%$ \\
\hline$\leqslant 60$ & 8 & 6.20 & 6 & 4.65 & 14 & 5.43 \\
\hline $61-65$ & 33 & 25.58 & 21 & 16.28 & 54 & 20.93 \\
\hline $66-70$ & 36 & 27.91 & 27 & 20.93 & 63 & 24.42 \\
\hline $71-75$ & 22 & 17.05 & 36 & 27.91 & 58 & 22.48 \\
\hline $76-80$ & 19 & 14.73 & 27 & 20.93 & 46 & 17.83 \\
\hline$\geqslant 81$ & 11 & 8.53 & 12 & 9.30 & 23 & 8.91 \\
\hline total & 129 & 100.00 & 129 & 100.00 & 258 & 100.00 \\
\hline
\end{tabular}

Table 2. Age correlations concerning glucose and lipid metabolism in male AIS patients

\begin{tabular}{lllllll}
\hline & glucose & TC & HDL-C & LDL-C & \multicolumn{2}{l}{ triglycerides age } \\
\hline glucose & 1 & 0.078 & 0.008 & 0.178 & 0.125 & -0.021 \\
\hline TC & 0.078 & 1 & 0.106 & 0.542 & 0.335 & -0.043 \\
\hline HDL-C & 0.008 & 0.106 & 1 & -0.017 & -0.033 & -0.018 \\
\hline LDL-C & 0.178 & 0.542 & -0.017 & 1 & 0.112 & -0.149 \\
\hline triglycerides & 0.125 & 0.335 & -0.033 & 0.112 & 1 & -0.121 \\
\hline age & -0.021 & -0.043 & -0.018 & -0.149 & -0.121 & 1 \\
\hline
\end{tabular}


In the male and female patients, Pearson's coefficient was highest between TC and LDL-C. This coefficient was followed by that between TC and triglycerides in the male patients and by that between glucose and LDL-C in the female ones. The male patients with normal triglyceride values and the female patients with reduced ones prevailed considerably, followed by the female patients with higher triglyceride values and the male patients with reduced ones. The difference between the mean LDL-C concentrations of the males and females aged $\geqslant 81$ years was statistically reliable $(t=2.82 ; \mathrm{p}<0.01)$.

Table 4 demonstrates the correlation dependences between the laboratory parameters of glucose, lipid and protein metabolism in our AIS patients. Pearson's correlation coefficient was highest between the variables 'TC' and 'LDL$\mathrm{C}^{\prime}$ but moderate - between some of the rest variables such as 'creatinine' and 'urea'; 'TC' and 'triglycerides', 'TC' and 'urea'; 'triglycerides' and 'urea', and 'LDL-C' and 'urea'.

\section{Discussion}

There were relatively weak, age-dependent correlations between mean glucose and LDL-C concentrations in females and males with AIS as well. The mean TC levels correlated strongly with LDL-C levels and moderately with triglyceride ones in males while in females, there was a moderate correlation between $\mathrm{TC}$ and LDL-C levels only. As a whole, the values of these laboratory parameters decreased with age.

Comprehensive analysis of the primary parameters of lipid metabolism revealed the presence of hyperlipidemia and dyslipidemia as well, thus convincingly proving the importance of these disturbances for the etiopathogenesis of AIS in advanced age.

The role of the metabolic abnormalities for AIS has also been discussed in some recent publications. Of 101 IS patients, 53 (52.48\% of the cases) had persistent impaired glucose tolerance or progression to diabetes mellitus [2]. They were older and more often had arterial hypertension and used statins. A model including age, current smoking, statin use, triglycerides, arterial hypertension, previous ischemic cardiovascular disease, body mass index, and fasting plasma glucose accurately predicted this glucose tolerance, with statin use, triglycerides, and fasting plasma glucose as the most important predictors. Important predictors and modifiable

Table 3. Age correlations concerning glucose and lipid metabolism in female AIS patients

\begin{tabular}{lllllll}
\hline & glucose & TC & HDL-C & LDL-C & triglycerides & age \\
\hline glucose & 1 & 0.112 & 0.132 & 0.228 & 0.029 & -0.048 \\
\hline TC & 0.112 & 1 & 0.020 & 0.346 & 0.102 & -0.070 \\
\hline HDL-C & 0.132 & 0.020 & 1 & 0.155 & 0.007 & -0.001 \\
\hline LDL-C & 0.228 & 0.346 & 0.155 & 1 & 0.000 & -0.121 \\
\hline triglycerides & 0.029 & 0.102 & 0.007 & 0.000 & 1 & -0.079 \\
\hline age & -0.048 & -0.070 & -0.001 & -0.121 & -0.079 & 1 \\
\hline
\end{tabular}

Table 4. Correlations between the laboratory parameters of glucose, lipid and protein metabolism in AIS patients

\begin{tabular}{llllllll}
\hline & glucose & TC & HDL-C & LDL-C & triglycerides & creatinine & urea \\
\hline glucose & 1 & 0.100 & -0.044 & 0.182 & 0.027 & 0.135 & 0.186 \\
\hline TC & 0.100 & 1 & 0.102 & 0.797 & 0.565 & 0.202 & 0.428 \\
\hline HDL-C & -0.044 & 0.102 & 1 & 0.099 & -0.247 & -0.125 & -0.189 \\
\hline LDL-C & 0.182 & 0.797 & 0.099 & 1 & 0.285 & 0.115 & 0.301 \\
\hline triglycerides & 0.027 & 0.565 & -0.247 & 0.285 & 1 & 0.226 & 0.370 \\
\hline creatinine & 0.135 & 0.202 & -0.125 & 0.115 & 0.226 & 1 & 0.575 \\
\hline urea & 0.186 & 0.428 & -0.189 & 0.301 & 0.370 & 0.575 & 1
\end{tabular}


barriers to lipid testing in pediatric IS in the USA, Australia and Chile such as age, sex, race, ethnicity, body mass index, other stroke risk factors, country, and recurrent thrombosis were identified [3].

A total of 5,151 Japanese individuals aged 3079 years were classified into six groups according to $\mathrm{LDL}-\mathrm{C}(<140$ and $\geqslant 140 \mathrm{mg} / \mathrm{dL}$ or lipid medication) and blood pressure (optimal, prehypertension, and arterial hypertension) levels [4]. There were 215 IS events during 13 years of follow-up. Hazard ratios for IS did not differ between normal and high LDL-C levels at the same blood pressure level.

The investigation of the fasting lipid profile in 90 IS patients and 101 healthy controls demonstrated a statistically significant association $(p<0.001)$ between the parameters of lipid profile of IS patients and controls as well as with IS prognosis [5]. The comparative study of lipid profiles among 324 IS Hispanic patients from Mexico and 236 ones from Miami showed that Mexicans had lower TC $(169.9 \pm 46.1$ versus $179.9 \pm 48.4 \mathrm{mg} / \mathrm{dL})$ and LDL $(92.3 \pm 7.1$ versus $108.2 \pm 40.8 \mathrm{mg} / \mathrm{dL}$ ), as well as higher triglyceride levels $(166.9 \pm 123.9$ versus $149.2 \pm 115.2 \mathrm{mg} / \mathrm{dL})$ [6]. These differences remained significant after adjusting for age, gender, arterial hypertension, diabetes mellitus, body mass index, smoking, IS subtype, and statin use.

Data on 27,493 participants from 3 population-based cohort studies: the Atherosclerosis Risk in Communities Study (median age of 54 years, median follow-up 20.7 years), the Rotterdam Study (median age of 68 years, median follow-up 14.3 years) and the Cardiovascular Health Study (median age of 71 years, median follow-up 12.8 years) displayed 2,559 IS events [7]. Basic models included established non-laboratory risk factors as well as diastolic blood pressure, TC/HDL-C ratio, body mass index, waist-to-hip ratio, and glomerular filtration rate. The high TC/HDL-C ratio increased IS rates. The cumulative incidence functions' performances were cross-validated by Harrell $\mathrm{C}$ statistics that increased more by model extension in the Atherosclerosis Risk in Communities and Cardiovascular Health Study cohorts.

A total of 1,059 IS patients from five community health centres in China were examined [8]. The proportions of IS patients with high $(\geqslant 1.55 \mathrm{mmol} / \mathrm{L})$, moderate (1.04-1.54 $\mathrm{mmol} / \mathrm{L})$ and low $(<1.04 \mathrm{mmol} / \mathrm{L})$ HDL-C levels were $15.58 \%, 54.58 \%$ and $29.84 \%$, respectively. The risk of IS recurrence increased with decreasing HDL-C level or HDL-C/TC ratio.

A definite cause of IS was identified in 202 out of 400 patients aged 16-54 years at a mean age of $44.5 \pm 8.5$ years [9]. These causes were classified using the ASCO (A for atherothrombosis, S for small vessel disease, $\mathrm{C}$ for cardiac source, $\mathrm{O}$ for other cause) classification system and included atherothrombosis (72 patients), cardioembolism (37 patients), small vessel disease (28 patients), other (65, including 44 patients with carotid or vertebral artery dissection). Atherothrombosis was associated with age, smoking, diabetes mellitus, arterial hypertension and low HDL-C; small vessel disease - with age and arterial hypertension while cardioembolism - with age only.

A total of 2,452 community-dwelling Japanese subjects aged $\geqslant 40$ years were prospectively followed-up for 24 years [10]. The age- and sex-adjusted incidence of lacunar infarction significantly increased with elevating non-HDL-C levels ( $\mathrm{p}$ for trend $<0.01$ ). This tendency was observed for atherothrombotic infarction ( $\mathrm{p}$ for trend $=0.098$ ), while for cardioembolic infarction ( $\mathrm{p}$ for trend $=0.007$ ), there was a significant inverse association. After adjustment for age, sex, diabetes mellitus, body mass index, systolic blood pressure, ECG abnormalities, regular exercise, current drinking and smoking, the associations remained significant for atherothrombotic infarction (adjusted hazard ratio 1.39, 95\% CI 1.09-1.79) and cardioembolic one (adjusted hazard ratio $0.64,95 \% \mathrm{CI}=0.47-0.85$ ).

The investigation of 149 patients, of which 61 - without carotid stenosis but 30 - with mild, 34 with moderate and 24 - with severe carotid stenosis showed that small, dense LDL-C (sdLDL-C) levels increased significantly with increasing stenosis severity $(0.54 \pm 0.19$, $0.71 \pm 0.18,0.98 \pm 0.19$ and $1.32 \pm 0.17 \mathrm{mmol} / \mathrm{L}$, respectively) [11]. Male sex, body mass index, diabetes mellitus, arterial hypertension, hyperlipidemia, drinking, smoking, and sdLDLC levels positively correlated with carotid stenosis. Levels of sdLDL-C were an independent risk factor of carotid stenosis $(p=0.041)$ and ischemic cerebral infarction in elderly patients.

The effect of dyslipidemia on outcome in 1,568 AIS patients in four hospitals in Shandong Province, China, was analyzed by multivariate 
logistic regression and propensity score-adjusted analysis [12]. The serum levels of TC, LDL-C, and HDL-C were significantly positively related with these patients' outcome.

Data from the medical records of 3,290 AIS admissions at a regional stroke centre in the USA within racial/ethnic groups revealed that South Asian and Hispanic men had a higher proportion of IS than women, while the opposite was true for Whites and African Americans ( $p=0.0014)$ [13]. All women, except South Asian ones, had higher mean plasma TC and higher blood circulating LDL levels $(\geqslant 100 \mathrm{mg} / \mathrm{dL})$ than men. We too established higher mean concentrations of TC and LDL-C in females than in males. On the other hand, there was a statistically significant difference between males and females aged $\geqslant 81$ years in terms of mean LDL-C levels.

Low cholesterol concentration in 190 IS patients was associated with older age, lower blood pressure, presence of angina pectoris, and higher risk of death [14]. Three-month, one- and five-year survival rates were $100 \%, 98 \%$, and $84 \%$, respectively, in high-cholesterol patients when compared with $92 \%, 87 \%$, and $57 \%$ in lowcholesterol ones $(p=0.0001$ with the log-rank test). Mortality risk increased for patients with low cholesterol (hazard ratio 1.97; 95\% CI 1.053.69) after adjustment for age and admission National Institutes of Health Stroke Scale score.

In a prospective observational study of 83 AIS patients at a median age of 76 years followed up for 28-30 months, triglycerides were considered as a continuous and a binary variable [15]. Higher triglycerides (default adjustments: stroke type, severity at presentation, age, atrial fibrillation, pre- and postindex event antiplatelet use, infarction volume, statin and ACE inhibitor use, on-admission fasting cholesterol, mean platelet volume, and glomerular filtration rate) were independently related to lower risk of all-cause mortality - hazard ratio 0.47 ; $95 \%$ CI $0.23-0.96$ and hazard ratio $0.45 ; 95 \% \mathrm{CI} \quad 0.21-0.98$, respectively.

\section{Conclusions}

In conclusion, metabolic disorders are relatively common among AIS patients and play a specific role as risk factors for this severe disease. These parameters should regularly be controlled within the outpatient clinical practice in order to warrant an effective AIS prevention.

\section{References}

1. Lisak M, Demarin V, Trkanjec Z, Basić-Kes V. Hypertriglyceridemia as a possible independent risk factor for stroke. Acta Clin Croat. 2013;52(4):458-63.

2. Fonville S, den Hertog HM, Zandbergen AA, Koudstaal PJ, Lingsma HF. Occurrence and predictors of persistent impaired glucose tolerance after acute ischemic stroke or transient ischemic attack. J Stroke Cerebrovasc Dis. 2014;23(6):1669-75.

3. Sultan S, Schupf N, Dowling M, DeVeber G, Kirton A, Elkind MS; IPSS Investigators. Predictors of cholesterol and lipoprotein(a) testing in children with arterial ischemic stroke. J Stroke Cerebrovasc Dis. 2014;23(9):2405-13.

4. Tsukinoki R, Okamura T, Watanabe M, Kokubo Y, Higashiyama A, Nishimura $K$, et al. Blood pressure, low-density lipoprotein cholesterol, and incidences of coronary artery disease and ischemic stroke in Japanese: the Suita Study. Am J Hypertens. 2014;27(11):1362-9.

5. Bharosay A, Bharosay VV, Bandyopadhyay D, Sodani A, Varma M, Baruah H. Effect of lipid profile upon prognosis in ischemic and haemorrhagic cerebrovascular stroke. Indian $\mathrm{J}$ Clin Biochem. 2014;29(3):372-6.

6. Arauz A, Romano JG, Ruiz-Franco A, Shang T, Dong $\mathrm{C}$, Rundek $\mathrm{T}$, et al. Differences in lipid profiles in two Hispanic ischemic stroke populations. Int J Stroke. 2014;9(4):394-9.

7. Ferket BS, van Kempen BJ, Wieberdink RG, Steyerberg EW, Koudstaal PJ, Hofman A, et al. Separate prediction of intracerebral hemorrhage and ischemic stroke. Neurology. 2014; 82(20):1804-12.

8. Mei L, Fang X, Mu L, Liu H, Zhang H, Qin X, et al. Association of serum high-density lipoprotein cholesterol level and risk of recurrent ischemic stroke. Zhonghua Xin Xue Guan Bing Za Zhi. 2014;42(4):295-300. Chinese.

9. Jaffre A, Ruidavets JB, Calviere L, Viguier A, Ferrieres J, Larrue V. Risk factor profile by etiological subtype of ischemic stroke in the young. Clin Neurol Neurosurg. 2014;120:78-83.

10. Imamura T, Doi Y, Ninomiya T, Hata J, Nagata M, Ikeda F, et al. Non-high-density lipoprotein cholesterol and the development of coronary heart disease and stroke subtypes in a general Japanese population: the Hisayama Study. Atherosclerosis. 2014;233(2):343-8.

11. Shen H, Zhou J, Shen G, Yang H, Lu Z, Wang H. Correlation between serum levels of small, dense low-density lipoprotein cholesterol and carotid stenosis in cerebral infarction patients $>65$ years of age. Ann Vasc Surg. 2014;28(2):375-80.

12. Xu T, Zhang JT, Yang M, Zhang H, Liu WQ, Kong $\mathrm{Y}$, et al. Dyslipidemia and outcome in patients with acute ischemic stroke. Biomed Environ Sci. 
— Arabadzhieva D., et al. Metabolic disturbances in the patients with acute ischemic stroke

2014;27(2):106-10.

13. Gezmu T, Schneider D, Demissie K, Lin Y, Giordano C, Gizzi MS. Lipid profiles and ischemic stroke risk: variations by sex within racial/ethnic groups. Int $\mathrm{J}$ Womens Health. 2014;6:585-95.

14. Markaki I, Nilsson U, Kostulas K, Sjöstrand C. High cholesterol levels are associated with improved long-term survival after acute ischemic stroke. J Stroke Cerebrovasc Dis. 2014;23(1):e4753.

15. Pikija S, Trkulja V, Juvan L, Ivanec M, Dukši D. Higher on-admission serum triglycerides predict less severe disability and lower all-cause mortality after acute ischemic stroke. J Stroke Cerebrovasc Dis. 2013;22(7):e15-24. 